Topic Introduction

\title{
Spinning-Disk Microscopy Systems
}

\author{
Tony Wilson
}

\section{INTRODUCTION}

The popularity of the confocal microscope in life science laboratories around the world is undoubtedly due to its ability to permit volume objects to be imaged and to be rendered in three dimensions. It is important to realize that the confocal microscope itself does not produce three-dimensional images. Indeed, it does the opposite. The critical property that the confocal microscope possesses, which the conventional microscope does not, is its ability to image efficiently (and in-focus) only those regions of a volume specimen that lie within a thin section in the focal region of the microscope. In other words, it is able to reject (i.e., vastly attenuate) light originating from out-of-focus regions of the specimen. To image a three-dimensional volume of a thick specimen, it is necessary to take a whole series of such thin optical sections as the specimen is moved axially through the focal region. Once this through-focus series of optically sectioned images has been recorded, it is a matter of computer processing to decide how the three-dimensional information is to be presented. There are many methods for producing optical sections, of which the confocal optical system is just one. This article reviews these methods and describes a number of convenient methods of implementation that can lead to, among other things, real-time image formation.

\section{OPTICAL SECTIONING}

In the following discussion, attention is restricted to bright-field or (single-photon) fluorescence imaging in which the optical sectioning results from the optical system of the microscope rather than any nonlinear interaction between the probe light and the specimen. To be able to make general remarks about various optical systems, the design is described in terms of the structure of the illumination and in terms of the structure of the detection. To put these terms in context, it is noted that, in the conventional fluorescence microscope, the specimen is illuminated uniformly and the fluorescence emitted by the specimen is imaged to an image plane in which the image intensity is viewed either directly by eye or via a charge-coupled device (CCD) camera. In this case, the structure of the illumination is uniform, as is the structure of the detection, and the microscope does not show optical sectioning. In the confocal microscope, on the other hand, point illumination and point detection are used to introduce optical sectioning. The optical principle can be seen in Figure 1, where the action of the point detector is to block light that originates in out-of-focus regions from passing through the pinhole (for an early description of confocal microscopy, see Wilson and Sheppard 1984; for a review of confocal microscopy methods and applications, see Wilson 1990). Its efficacy in achieving this, which also determines the axial width of the optical section, clearly depends on the size and the shape of the pinhole used. An infinitely large pinhole, for example, would block no light and, hence, provide no optical sectioning. This effect is discussed in detail elsewhere (Wilson 1989, 1995; Wilson and Carlini 1987). The system illustrated in Figure 1 might be regarded as the ultimate in structured illumination and detection-point illumination and point detection-and has resulted in the desired optical sectioning but has only produced an image of a single point of the specimen.

To produce an image of a finite region of the specimen, it is necessary to introduce scanning so as to probe the entire specimen. In general terms, a particular structure has been introduced to both the illumination and the detection, which might also be referred to as modulation, such that the optical system shows optical sectioning. Any undesirable side effects of this modulation must then be removed to obtain the desired image. In this particular case, the modulation results in a restriction

Adapted from Imaging: A Laboratory Manual (ed. Yuste). CSHL Press, Cold Spring Harbor, NY, USA, 2010.

Cite as: Cold Spring Harb Protoc; 2010; doi:10.1101/pdb.top88

www.cshprotocols.org 


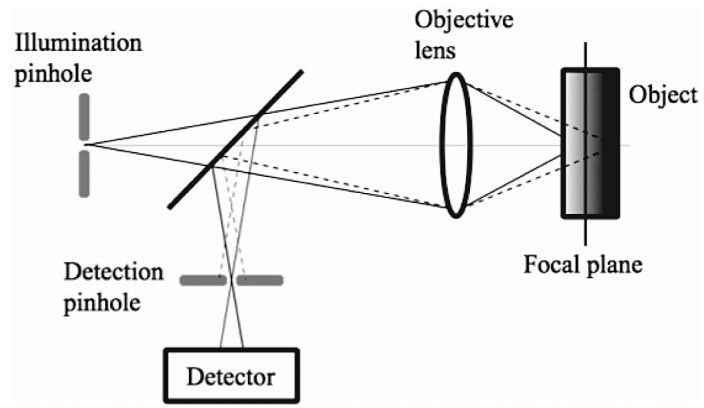

FIGURE 1. The physical origin of the depth discrimination or optical sectioning property of the confocal optical system. A barrier in the form of a pinhole aperture is used to physically prevent (block) light originating from regions in the specimen from passing through the pinhole and, hence, being detected by the photodetector and thus contributing to the final image contrast.

of the field of view to a single point; hence, a demodulation stage consisting of scanning is required to restore the field of view. Practical implementations of the demodulation are discussed below, but there are two basic approaches. In the first, a single point source-point detector confocal system is used together with a scanning mechanism designed to scan a single focused spot of light with respect to the specimen. In the second approach, a number of confocal systems are constructed in parallel. These serve to produce many focused spots of light, which are used to image different parts of the specimen simultaneously. This is achieved by using an aperture disk consisting of many pinholes.

Another way to think about optical sectioning is in terms of the way in which the spatial frequencies present in the specimen are imaged. In essence, we describe the fluorescence distribution within the specimen in terms of its spatial frequency spectrum (Fourier content) and ask how each of these spatial frequency components is imaged by the optical system. The optical transfer function of the optical system provides the answer to this question because it describes how efficiently each spatial frequency is imaged. A requirement that the system shows optical sectioning might be that the contrast of all spatial frequencies must attenuate as the microscope is defocused. Figure 2A shows the optical transfer function of a confocal fluorescence microscope, in which the contrast of all spatial frequencies attenuates with increasing defocus. Figure $2 \mathrm{~B}$, on the other hand, shows the equivalent function for the conventional fluorescence microscope. In this case, it is only the zero spatial frequency whose contrast does not attenuate with increasing defocus. The contrast of all other spatial frequencies is seen to reduce as the degree of defocus increases.

Although the aperture disk consisting of many pinholes was described above as a natural way to parallelize many confocal microscopes, it may also be thought of as acting like a mask that causes the whole specimen to be illuminated by a particular structure. It is natural, therefore, to ask whether there are other simple forms of structure to the illumination that may be used to introduce optical sectioning. If we modify the illumination system of the microscope so as to project a single spatial frequency grid pattern onto the object, the microscope will then image efficiently only that portion of the object where the grid pattern is in focus (Fig. 2B). We will thus obtain an optically sectioned image of the object but with the (unwanted) grid pattern superimposed. The rate of attenuation with defocus or optical sectioning strength will, of course, depend on the particular spatial frequency that is projected onto the object (Fig. 2B). For example, a 40- $\mathrm{mm}$ pitch grid imaged using a $63 \mathrm{X}, 1.4-\mathrm{NA}$
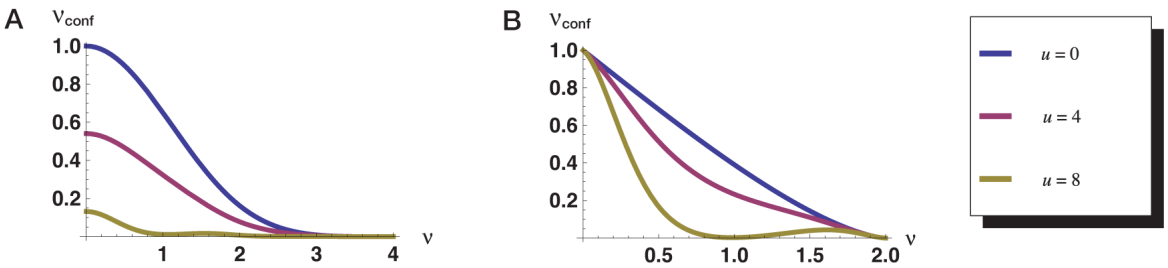

FIGURE 2. The confocal optical transfer function as a function of the normalized spatial frequency for a number of values of defocus $u(A)$. The normalized defocus $u$ is related to the actual defocus $z$ by $u=4 k n z \sin ^{2}(\alpha / 2)$ in which the numerical aperture (NA) is given by $\mathrm{NA}=n \sin (\alpha)$. The normalized spatial frequency $v$ is related to the actual spatial frequency $f$ measured in the focal plane via $v=f \lambda / N A$. We note that all spatial frequencies attenuate with increasing defocus. The optical transfer function of the conventional microscope as a function of the normalized spatial frequency for a number of values of defocus $u(B)$. Note that all spatial frequencies attenuate with increasing defocus apart from the zero spatial frequency case. (For color figure, see doi: 10.1101/pdb.top88 online at www.cshprotocols.org.) 
objective lens with light of wavelength $0.5 \mu \mathrm{m}$ yields $v=0.56$, whereas a $40-\mu \mathrm{m}$ pitch yields $v=0.28$ and a $20-\mu \mathrm{m}$ pitch grid gives $v=1.12$. Here we have used the structure of the illumination (harmonic modulation) to introduce optical sectioning. The price is that the optical section is now delineated or labeled by that portion of the image where the superimposed grid pattern is visible. It is now necessary to introduce a demodulation stage whereby the out-of-focus regions as well as the grid pattern are removed from the "raw" image to reveal the desired optically sectioned image. This may be done in two ways, computationally or optically. The computational approach typically requires that three raw images be taken, corresponding to three different spatial positions of the illumination grid. This is the approach taken in several commercial structured illumination systems such as the OptiGrid system from Qioptiq (Neil et al. 1997). The alternative optical demodulation technique, which will be discussed in this article, is to combine harmonic structured illumination with harmonic structured detection. In this case, an identical mask is used for both the illumination and detection. Demodulation is carried out by scanning the masks in synchronism.

It should be noted that a system with uniform structure of illumination and detection-the conventional microscope-does not exhibit optical sectioning, whereas one with point illumination and point detection-the confocal system—does exhibit optical sectioning. An equivalent way of saying this is that the conventional system employs zero spatial frequency illumination and detection whereas the point source/detector confocal system employs full spatial frequency illumination and detection. The harmonic approach just discussed, on the other hand, lies somewhere between these approaches, because only one spatial frequency is used for both the illumination and detection. The nature of the illumination/detection used in the last two cases requires that a further demodulation step-often achieved by scanning—-be performed to provide a full field optically sectioned image.

\section{PRACTICAL REALIZATION OF OPTICAL SECTIONING MICROSCOPES}

The practical implementation of these two approaches to achieve optical sectioning will now be discussed, beginning with the traditional confocal system.

It is clear from the previous discussion that an optical system consisting of a single point source and single point detector serves to discriminate against light originating from out-of-focus planes. Figure 1 shows the generic optical system. The light source is typically a laser, because traditional microscope illumination systems are insufficiently bright. A photomultiplier tube has usually been used as the photodetector. Because this system probes only one point of the specimen, scanning must be used to obtain an image of a whole optical section. This may be achieved in a variety of ways. The specimen may be physically scanned with respect to the fixed focal spot. Alternatively, the objective lens may also be scanned. These approaches have advantages from both the optical performance and optical design points of view but are generally considered to be impractical. In most commercial designs, therefore, the specimen is fixed and the scanning is achieved by scanning the focused spot of light across the fixed specimen by the use of galvonometer mirror scanners. This allows an optical section to be easily recorded. To record the next optical section, however, it is necessary to physically move the specimen axially to bring the next region into the focal volume of the confocal microscope. Commercial systems do not allow this important z-scanning step to be performed quickly, so this represents a bottleneck in the speed with which a through-focus set of images may be obtained. Recent work has shown that high-speed optical refocusing can be achieved, and hence this bottleneck may be removed (Botcherby et al. 2008, 2009). Although the layout of Figure 1 is typical, there are problems to be overcome relating to system alignment in the sense that the detector pinhole must be located in a position optically equivalent to the source pinhole. These problems may be resolved if a reciprocal geometry is employed in which the same pinhole is used both as source and detector pinhole. In practice, these systems are often more easily implemented when a single-mode optical fiber replaces the pinhole (Kimura and Wilson 1991). However, all of these approaches involve the use of one confocal optical system, and the image is obtained serially by the appropriate scanning of the spot in three dimensions with respect to the specimen.

An advantage may be gained by building an optical layout consisting of many confocal systems lying side by side. In this way, many parts of the specimen will be imaged confocally at the same time. This has the advantage of increasing image acquisition speed as well as dispensing with the need to use laser illumination. Each pinhole acts as both the illumination and detection pinhole and so the system acts rather like a large number of parallel, reciprocal geometry, confocal microscopes, each imaging a specific point on the object. However, it must be remembered that the confocal system achieves 
depth discrimination by blocking out-of-focus light reaching the image by the use of a limiting pinhole detector. This observation leads to the conclusion that the neighboring confocal systems must be placed sufficiently far apart that any out-of-focus light from one confocal system is not collected by an adjacent system. In other words, cross talk between neighboring confocal systems must be prevented. In practice, this means that the pinholes must be placed on the order of 10 times their diameters apart, which has two immediate consequences. First, only a small amount-typically $1 \%$-of the available light is used for imaging, and, second, the wide spacing of the pinholes means that the object is only sparsely probed. To probe-and hence image - the whole object, it is usual to arrange the pinhole apertures in a series of Archimedean spirals and to rotate the (Nipkow) disk. The generic layout of any system that is designed to contain many confocal systems operating in parallel is shown in Figure 3A. The original idea for such an approach goes back to Mojmir Petrán in the late 1960s (Petrán et al. 1968). A single-sided variant was subsequently introduced by Kino and his colleagues (Xiao and Kino 1987). The key element to these systems is a spinning Nipkow disk containing many pinholes. These systems are capable of producing high-quality images without the need to use laser illumination in real time at both television rate and higher imaging speeds. A further development, which does, however, require the use of laser illumination and, hence, restricts the use to fluorescence imaging, is to introduce an array of microlenses to concentrate the illumination laser light into the source pinholes (Ichihara et al. 1996).

One approach to make greater use of the available light is to place the pinholes closer together. However, this means that cross talk between the neighboring confocal systems inevitably occurs; hence, a method must be devised to prevent this. To achieve this goal, the Nipkow disk of the tandem-scanning microscope is replaced with an aperture mask consisting of many pinholes placed as close together as possible. This aperture mask has the property that any of its pinholes can be opened and closed independently of the others in any desired time sequence. This might be achieved, for example, by using a liquid-crystal spatial light modulator. Because we require there to be no cross talk between the many parallel confocal systems, it is necessary to use a sequence of openings and closings of each pinhole that is completely uncorrelated with the openings and the closings of all the other pinholes. There are many such orthonormal sequences available. However, they all require the use of both positive and negative numbers, and, unfortunately, we cannot have a negative intensity of light! The pinhole is either open, which corresponds to 1 , or closed, which corresponds to 0 . There is no position that can correspond to -1 . The way to avoid the dilemma is to obtain the confocal signal indirectly. To use a particular orthonormal sequence $b_{i}(t)$ of plus and minus $1 \mathrm{~s}$, for the ith pinhole, we must add a constant offset to the desired sequence to make a sequence of positive numbers, which can be encoded in terms of pinhole opening and closing. Thus, we encode each of the pinhole openings and closings as $\left(1+b_{i}(t)\right) / 2$, which will correspond to open $(1)$ when $b_{i}(t)=1$ and to close $(0)$ when $b_{i}(t)$ $=-1$. The effect of adding the constant offset to the desired sequence is to produce a composite image that will be partly confocal because of the $b_{i}(t)$ terms and partly conventional because of the constant term. The method of operation is now clear. We first take an image with the pinholes encoded as we have just discussed and so obtain a composite conventional plus confocal image. We then switch all the pinholes to the open state to obtain a conventional image. It is then a simple matter to subtract the two images in real time using a computer to produce the confocal image.

Although this approach may be implemented using a liquid-crystal spatial light modulator, it is cheaper and simpler merely to impress the correlation codes photolithographically on a disk and to rotate the disk so that the transmissivity at any picture point varies according to the desired orthonormal sequence. A blank sector may be used to provide the conventional image. If this approach is adopted, then all that is required is to replace the single-sided Nipkow disk of the tandem-scanning microscope with a suitably encoded aperture disk (Juskaitis et al. 1996; Wilson et al. 1996). The coded sector on the disk may be coded so as to provide the appropriate correlation codes, or, alternatively, it may consist of a pattern of grid lines to simulate the harmonic illumination/detection case (Neil et al. 1998).

\section{Double-Sided Operation}

We have seen that the image obtained from the coded sector of the disk is a composite image from which the conventional image needs to be removed. If, rather than use a blank sector, we were to encode the whole disk such that the image we obtained may be written as $I_{1}=I_{\text {conv }}+I_{\text {conf }}$, we would need to find another way to remove the conventional image. One approach is suggested in Figure $3 \mathrm{~B}$, in which a second light source is used. In this case, a composite image of the form $I_{2}=I_{\text {conv }}-I_{\text {conf }}$ is 

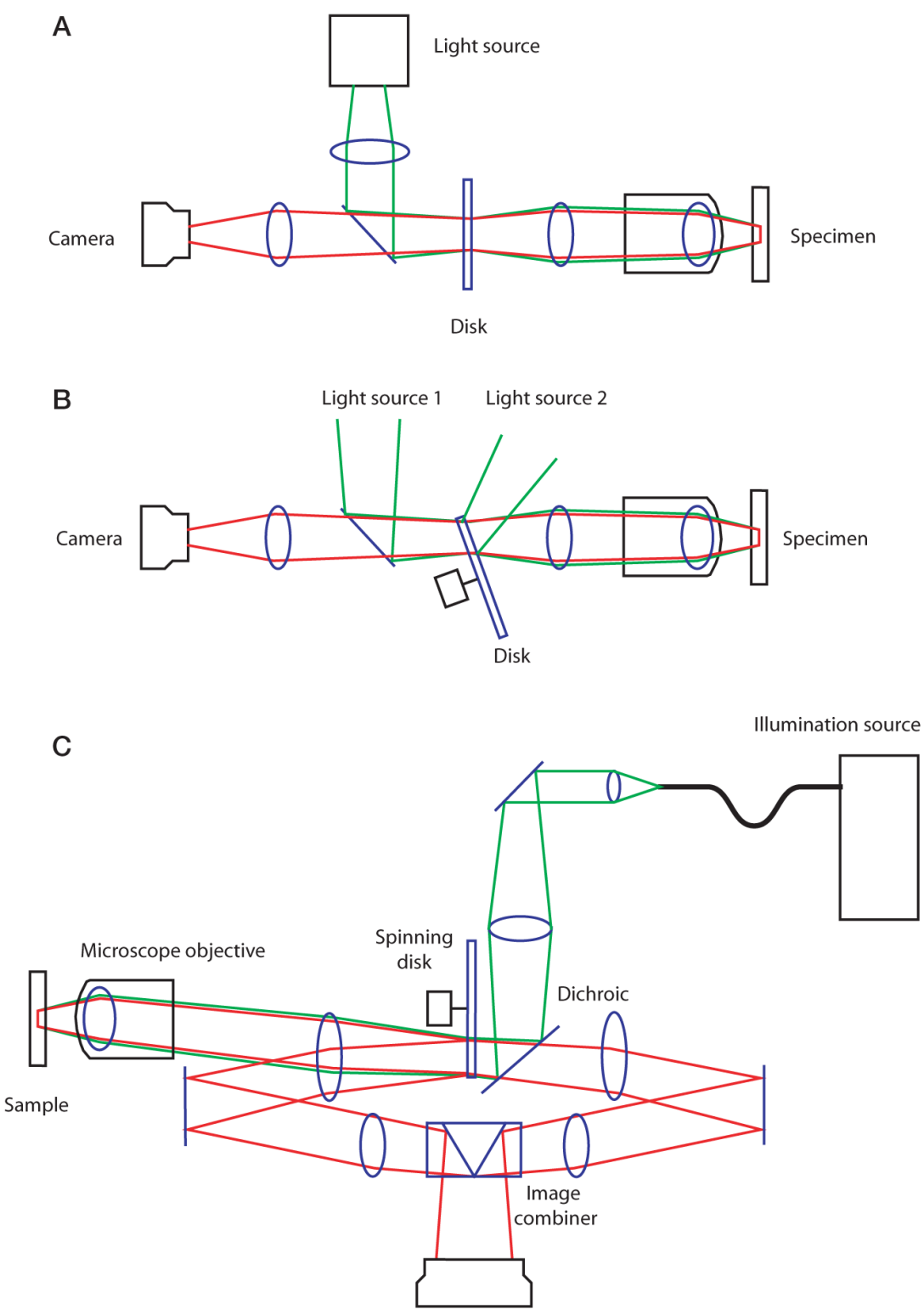

FIGURE 3. (A) The generic form of a spinning-disk-based microscope system. In the case of the tandem-scanning microscope, the disk consists of a large number of pinholes, each of which acts as a reciprocal geometry confocal system. The pinholes, which are placed far apart to prevent cross talk between adjacent confocal systems, are usually arranged in an Archimedean spiral on a Nipkow disk. Rotation of the disk causes the entire specimen to be imaged. In the case of the correlation code microscope, the appropriate codes are impressed photolithographically onto the disk, which is then rotated to effect the desired time-dependent correlation codes. In the harmonic illumination and detection case, a stripe pattern is imposed on the disk. In this case, the disk is spun to achieve the desired demodulation. (B) An example of a double-sided operation in which two light sources are used to sequentially generate the two composite images: conventional plus confocal and conventional minus confocal. (C) An optical configuration that permits both composite images to be recorded simultaneously, thereby removing any motion artifacts. (For color figure, see doi: 10.1101/ pdb.top88 online at www.cshprotocols.org.)

obtained. It is clear that a confocal image $I_{1}-I_{2}=2 I_{\text {conf }}$ may be readily extracted with a more efficient use of light than in the single-sided disk case in which $I_{1}$ and $I_{\text {conv }}$ are obtained sequentially.

Although such an approach is entirely feasible, it does require extremely careful design so as to provide equivalent uniform illumination at each flip of the mirror. A preferred approach might be to use a single light source and a single camera. The optical 

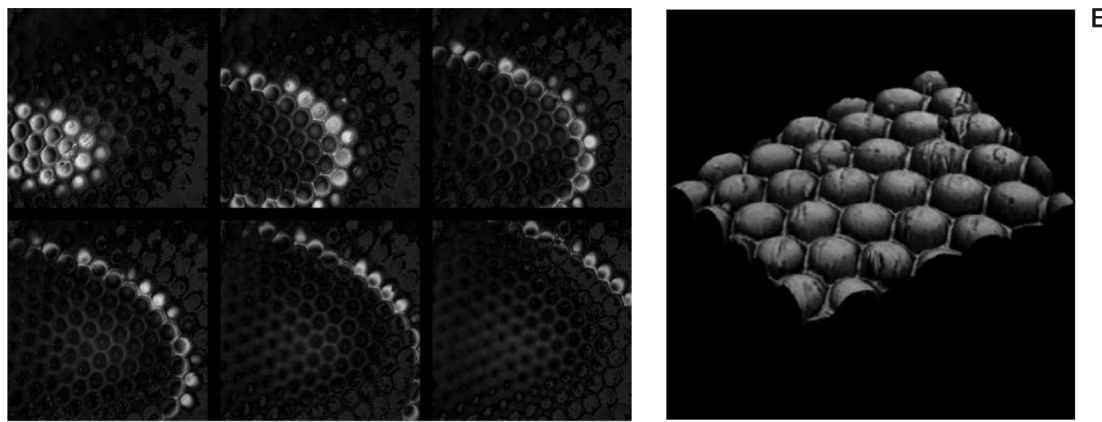

FIGURE 4. (A) A through-focus series of images taken of the eye of a fly. (B) A three-dimensional rendering of the eye of the fly constructed from the through-focus series of images presented in $A$.

system (Fig. 3C) would be such that the camera recorded the two required images simultaneously, thus eliminating any possibility of motion and other artifacts between the capture of the two required raw images. These systems operate well with standard microscope illuminators (e.g., EXFO Inc.) and standard CCD cameras, producing images that are directly comparable to those taken with traditional laser-based confocal systems. Figure 4 shows a typical through-focus series of images together with a standard three-dimensional rendering.

\section{CONCLUSION}

The confocal microscope is now firmly established as a workhorse instrument in laboratories throughout the world because of its ability to enable volume specimens to be imaged in three dimensions. However, there is still much work to be performed to make these instruments suitable for high-speed imaging of living specimens. Further advances will require a combination of new contrast mechanisms together with advances in instrument design. This article has described a parallelization of the traditional confocal principle so as to permit real-time confocal imaging without the need for laser illumination. The concept of structured illumination and structured detection has been introduced to stimulate the search for alternative methods of image encoding to reveal optical sectioning.

\section{REFERENCES}

Botcherby EJ, Booth MJ, Juskaitis R, Wilson T. 2008. Real-time extended depth of field microscopy. Opt Express 16: 21843 21848.

Botcherby EJ, Booth MJ, Juskaitis R, Wilson T. 2009. Real-time slit scanning microscopy in the meridional plane. Opt Lett 34: 15041506.

Ichihara A, Tanaami T, Isozaki K, Sugiyama Y, Kosugi Y, Mikuriya K, Abe M, Uemura I. 1996. High-speed confocal fluorescence microscopy using a Nipkow scanner with microlenses for 3-D imaging of single fluorescent molecule in real-time. Bioimages 4: 57-62.

Juskaitis R, Wilson T, Neil MA, Kozubek M. 1996. Efficient real-time confocal microscopy with white light sources. Nature 383: 804-806.

Kimura S, Wilson T. 1991. Confocal scanning optical microscope using single-mode fiber for signal detection. Appl Opt 30: 2143 2150.

Neil MA, Juskaitis R, Wilson T. 1997. Method of obtaining optical sectioning by using structured light in a conventional micro- scope. Opt Lett 22: 1905-1907.

Neil MAA, Wilson T, Juskaitis R. 1998. A light efficient optically sectioning microscope. J Microsc 189: 114-117.

Petrán M, Hadravsky M, Egger MD, Galambos R. 1968. Tandemscanning reflected-light microscope. J Opt Soc Am 58: 661-664.

Wilson T. 1989. Optical sectioning in confocal fluorescent microscopes. J Microsc 154: 143-156.

Wilson T. 1990. Confocal microscopy. Academic, London.

Wilson T. 1995. The role of the pinhole in confocal imaging system. In Handbook of biological confocal microscopy, 2nd ed. (ed. JB Pawley), pp. 167-182. Plenum, New York.

Wilson T, Carlini AR. 1987. Size of the detector in confocal imaging systems. Opt Lett 12: 227-229.

Wilson T, Sheppard CJR. 1984. Theory and practice of scanning optical microscopy. Academic, London.

Wilson T, Juskaitis R, Neil MA, Kozubek M. 1996. Confocal microscopy by aperture correlation. Opt Lett 21: 1879-1881.

Xiao GQ, Kino GS. 1987. A real-time scanning optical microscope. Proc SPIE 809: 107-113. 


\section{Spinning-Disk Microscopy Systems}

Tony Wilson

Cold Spring Harb Protoc; doi: 10.1101/pdb.top88

\begin{tabular}{|c|c|}
\hline $\begin{array}{r}\text { Email Alerting } \\
\text { Service }\end{array}$ & Receive free email alerts when new articles cite this article - click here. \\
\hline $\begin{array}{l}\text { Subject } \\
\text { Categories }\end{array}$ & $\begin{array}{l}\text { Browse articles on similar topics from Cold Spring Harbor Protocols. } \\
\text { Cell Imaging (525 articles) } \\
\text { Confocal Microscopy (114 articles) } \\
\text { Fluorescence (517 articles) } \\
\text { Fluorescence, general ( } 341 \text { articles) } \\
\text { Imaging Development (255 articles) } \\
\text { Imaging for Neuroscience (342 articles) } \\
\text { Imaging/Microscopy, general (579 articles) } \\
\text { In Vivo Imaging (334 articles) } \\
\text { In Vivo Imaging, general (168 articles) } \\
\text { Video Imaging / Time Lapse Imaging (171 articles) } \\
\text { Visualization (524 articles) } \\
\text { Visualization, general (369 articles) }\end{array}$ \\
\hline
\end{tabular}

